\title{
Optimal Broadcasting in Even Tori with Dynamic Faults
}

\author{
Stefan Dobrev and Imrich Vrt'o* \\ Institute of Mathematics, Slovak Academy of Sciences \\ Department of Informatics, P.O.Box 56, 84000 Bratislava, Slovak Republic \\ \{kaifdobr, vrto\}@savba.sk
}

\begin{abstract}
We consider a broadcasting problem in the $n$-dimensional $k$-ary even torus in the shouting communication mode, i.e. any node of a network can inform all its neighbours in one time step. In addition, during any time step a number of links of the network can be faulty. Moreover the faults are dynamic. The problem is to determine the minimum broadcasting time if at most $2 n-1$ faults are allowed in any step. In [3], it was shown that the broadcasting time is at most diameter $+O(1)$, provided that $k$ is limited by a polynomial of $n$. In our paper we drop this additional assumption and prove that the broadcasting can be always done in time diameter +2 . The bound is the best possible.
\end{abstract}

\section{Introduction}

Broadcasting is the standard communication problem in interconnection networks when a node has to send a message to all other nodes. There are many applications of the broadcasting problem in parallel and distributed computing [6,8,9]. Recently, a lot of attention has been paid to fault-tolerant dissemination of information in networks [10. In this paper we consider the shouting communication mode in which any node can inform all its neighbours in one time step. In addition, we assume that during any time step a number of links of the network can be faulty. This model was introduced by Santoro and Widmayer [11]. The problem is to determine the minimum broadcasting time if at most $f$ faults are allowed in any time step, where $f$ stands for the edge connectivity minus 1 of the network. For the hypercube, the problem was studied in 3710] and completely solved in [5]. For the $n$-dimensional general torus, Chlebus, Diks and Pelc [2] proved an upper bound on the brodcasting time $O$ (diameter). De Marco and Rescigno [4] showed for the $n$-dimensional $k$-ary even torus that the broadcasting time is at most diameter $+O(1)$, provided that $k$ is limited by a polynomial of $n$. In our paper we drop this additional assumption and prove that the broadcasting can be always done in time diameter +2 . The bound is the best possible.

Our method previously used in [5] is related to the isoperimetric problem in graphs and can be applied to other networks.

\footnotetext{
^ Supported by the VEGA grant No. 2/7007/20.
} 


\section{Model and Basic Facts}

Let $C_{n, k}$ be a network of processors connected as the $n$-dimensional $k$-ary torus defined as the cartesian product of $n$ cycles $C_{k}$, for $k$ even. The network $C_{n, k}$ has $k^{n}$ nodes, is regular of degree $2 n$, with edge connectivity $2 n-1$. Its diameter equals $n k / 2$. The links are bidirectional. The computation is synchronous. In one time step a node is able to send its message to all its neighbours. This is called the shouting mode. In each time step at most $2 n-1$ links are faulty, i.e., the message transmitted along the faulty link is not delivered. The faults are dynamic in the sense that the set of faulty links can change during the execution of the broadcast. Initially, a node of $C_{n, k}$ knows a message. This message needs to be sent to all other nodes. Our problem is to determine the minimum time to broadcast the message in the torus $C_{n, k}$, provided that the faults are distributed in the worst possible manner.

The vertices of $C_{n, k}$ are $\{-k / 2,-k / 2+1, \ldots, k / 2-1\}$, and two vertices $u$ and $v$ are adjacent if $|u-v|(\bmod k)=1$. The vertices of $C_{n, k}$ are $n$-tuples $\left(x_{1}, x_{2}, \ldots, x_{n}\right)$, where $-k / 2 \leq x_{i} \leq k / 2-1$, for $i=1,2, \ldots, n$. Define $\mathbf{o}=$ $(0,0, \ldots, 0)$. Let $d(u, v)$ be the distance between $u$ and $v$ in $C_{n, k}$. Denote $S(r)=$ $\left\{v \in C_{n, k} \mid d(v, \mathbf{o})=r\right\}$ and $V(r)=\left\{v \in T_{n, k} \mid d(v, \mathbf{o}) \leq r\right\}=\cup_{i=0}^{r} S(r)$. Clearly, $|S(r)|$ is the number of integer solutions of the equation $\left|x_{1}\right|+\left|x_{2}\right|+\ldots+\left|x_{n}\right|=r$, where $-k / 2 \leq x_{r} \leq k / 2-1$. Since $k$ is even we have $|S(r)|=|S(n k / 2-r)|$, which implies $|V(r)|+|V(n k / 2-r-1)|=k^{n}$. Moreover, $|S(0)|=1,|S(1)|=2 n$ and $|S(2)|=2 n^{2}$, for $k \geq 6$.

Let $A$ be a subset of vertices of $C_{n, k}$. Let $\partial(A)$ denote the set of all vertices in distance at most 1 from $A$ in $C_{n, k}$. Bollobás and Leader [1] proved:

Lemma 1. Let $C_{n, k}$ be the $n$-dimensional $k$-ary torus with $k$ even, and let $A$ be a nonempty subset of vertices of $C_{n, k}$. If $|A|=|V(r)|+\alpha|S(r+1)|$, for some $r$ and $0 \leq \alpha<1$, then $|\partial(A)| \geq|V(r+1)|+\alpha|S(r+2)|$.

\section{Optimal Upper Bound on the Broadcasting Time}

In this Section we use Lemma 1 to bound the broadcasting time. First we state a technical lemma, proof of which will appear in the full version.

Lemma 2. Denote

$$
X=1-\frac{3}{n}-\sum_{r=3}^{\left\lfloor\frac{n k}{4}\right\rfloor} \frac{4 n}{|S(r)|} .
$$

The value $X$ is nonnegative for $k \geq 6$ and $n \geq k+4$ or $k \geq n \geq 10$.

Theorem 1. Assume that $k \geq 6$ and $n \geq k+4$ or $k \geq n \geq 10$. The minimum broadcasting time $T$ in the $n$-dimensional $k$-ary torus, for $k$ even, with $2 n-1$ dynamic link faults satisfies $T \leq n k / 2+2$. The bound is the best possible. 
Proof. The broadcasting scheme is simple. Initially, the node o contains the message to be disseminated. In each time step each node sends the message to all its neighbours. The analysis follows. By $A_{m}$ we will denote suitable sets of nodes which know the message after the $m$-th time step. Observe that the number of nodes that know the message after the $m$-th step is at least $\left|\partial\left(A_{m-1}\right)\right|-(2 n-1)$. Clearly, there exist sets $A_{0}$ and $A_{1}$ and $A_{2}$ s.t. $\left|A_{0}\right|=1,\left|A_{1}\right|=2$ and $\left|A_{2}\right|=$ $1+2 n=|V(1)|$. According to Lemma $11\left|\partial\left(A_{2}\right)\right| \geq V(2) \mid$.

Because of the $2 n-1$ faulty links, we have

$$
\left|\partial\left(A_{2}\right)\right|-(2 n-1) \geq 2 n^{2}+2=|V(1)|+\alpha_{3}|S(2)|,
$$

where $\alpha_{3}=1-1 / n$. Define $A_{3}$ to be a subset of nodes that know the message after the 3-rd step and satisfies $\left|A_{3}\right|=|V(1)|+\alpha_{3}|S(2)|$. Similarly, $\left|\partial\left(A_{3}\right)\right| \geq$ $|V(2)|+\alpha_{3}|S(3)|$.

Because of the $2 n-1$ faulty links, we have

$$
\left|\partial\left(A_{3}\right)\right|-(2 n-1)=|V(2)|+\alpha_{3}|S(3)|-(2 n-1) \geq|V(2)|+\alpha_{4}|S(3)|,
$$

where $\alpha_{4}=\alpha_{3}-\frac{2 n}{|S(3)|}$. Define $A_{4}$ to be a subset of nodes that know the message after the 4-th step and satisfies $\left|A_{4}\right|=|V(2)|+\alpha_{4} \mid S(3 \mid$.

We prove by induction that for $4 \leq m \leq \frac{n k}{2}-1$

$$
\left|A_{m}\right|=|V(m-2)|+\alpha_{m}|S(m-1)|
$$

where

$$
\alpha_{m}=1-\frac{1}{n}-\frac{2 n}{|S(3)|}-\frac{2 n}{|S(4)|}-\ldots-\frac{2 n}{|S(m-1)|} .
$$

Assume the claim holds for some $4 \leq m-1 \leq \frac{n k}{2}-2$, i.e.

$$
\left|A_{m-1}\right|=|V(m-3)|+\alpha_{m-1}|S(m-2)|,
$$

where

$$
\alpha_{m-1}=1-\frac{1}{n}-\frac{2 n}{|S(3)|}-\frac{2 n}{|S(4)|}-\ldots-\frac{2 n}{|S(m-2)|} .
$$

Lemma 1 implies

$$
\begin{aligned}
\left|\partial\left(A_{m-1}\right)\right|-(2 n-1) & =|V(m-2)|+\alpha_{m-1}|S(m-1)|-(2 n-1) \\
& \geq|V(m-2)|+\alpha_{m}|S(m-1)|
\end{aligned}
$$

where $\alpha_{m}=\alpha_{m-1}-\frac{2 n}{\mid S(m-1)}$. Define $A_{m}$ to be a subset of nodes that know the message after the $m$-th step and satisfies $\left|A_{m}\right|=|V(m-2)|+\alpha_{m}|S(m-1)|$. Then

$$
\alpha_{m}=1-\frac{1}{n}-\sum_{i=3}^{m-1} \frac{2 n}{|S(i)|} .
$$

Now we use a dual argument. By $B_{m}$ we will denote suitable subsets of nodes, which do not know the message after the $m$-th step. Assume that after 
the $(n k / 2+2)$-nd step there exists at least one node which does not know the message. Observe that the number of nodes that do not know the message after the $(m-1)$-st step is at least $\left|\partial\left(B_{m}\right)\right|-(2 n-1)$. Clearly there exist sets $B_{\frac{n k}{2}+2}$, $B_{\frac{n k}{2}+1}$ and $B_{\frac{n k}{2}}$ such that $\left|B_{\frac{n k}{2}+2}\right|=1,\left|B_{\frac{n k}{2}+1}\right|=2$ and $\left|B_{\frac{n k}{2}}\right|=1+2 n$. Similarly as for $A_{3}$ we determine $\left|B_{\frac{n k}{2}-1}\right|=2 n^{2}+2$.

Now compute

$$
\begin{aligned}
\left|A_{\frac{n k}{2}-1}\right|+\left|B_{\frac{n k}{2}-1}\right| & =\left|V\left(\frac{n k}{2}-3\right)\right|+\left(1-\frac{1}{n}-\sum_{r=3}^{\frac{n k}{2}-2} \frac{2 n}{|S(r)|}\right)\left|S\left(\frac{n k}{2}-2\right)\right|+2 n^{2}+2 \\
& \geq k^{n}+1+2 n^{2}\left(1-\frac{3}{n}-\sum_{r=3}^{\left\lfloor\frac{n k}{4}\right\rfloor} \frac{4 n}{|S(r)|}\right) .
\end{aligned}
$$

By Lemma 2 the expression in the brackets is positive which implies a contradiction.

Finally, we show that there is a distribution of faults in each step which forces the brodcasting time $n k / 2+2$. Consider vertices $u, v$ such that $d(u, v)=n k / 2$. Let $u^{\prime} \quad\left(v^{\prime}\right)$ be a neighbor of $u(v)$. Initially, let $u^{\prime}$ knows the message. In the first step we place faults on all edges adjacent to $u^{\prime}$, except for $u^{\prime} u$. From now on we place faults on all edges adjacent to $v^{\prime}$, except for $v^{\prime} v$. After $n k / 2+1$ steps, the message reaches the vertex $v$ and one additional step is necessary to complete the broadcasting.

\section{References}

1. Bollobás, B., Leader, I., An isoperimetric inequality on the discrete torus, SIAM J. on Discrete Mathematics 3 (1990), 32-37.

2. Chlebus, B., Diks, K., Pelc, A., Broadcasting in synchronous networks with dynamic faults, Networks 27 (1996), 309-318.

3. De Marco, G., Vaccaro, U., Broadcasting in hypercubes and star graphs with dynamic faults, Information Processing Letters 66 (1998), 321-326.

4. De Marco, G., Rescigno, A.A., Tighter bounds on broadcasting in torus networks in presence of dynamic faults, Parallel Processing Letters, to appear.

5. Dobrev, S., Vrt'o, I., Optimal broadcasting in hypercubes with dynamic faults, Information Processing Letters 71 (1999), 81-85.

6. Fraigniaud, P., Lazard, E., Methods and problems of communication in usual networks, Discrete Applied Mathematics 53 (1994), 79-133.

7. Fraigniaud, P., Peyrat, C., Broadcasting in a hypercube when some calls fail, Information Processing Letters 39 (1991), 115-119.

8. Hedetniemi, S.M., Hedetniemi, S.T., and Liestman, A., A survey of gossiping and broadcasting in communication networks, Networks 18 (1986), 319-349.

9. Hromkovic, J., Klasing, R., Monien, B., Paine, R., Dissemination of information in interconnection networks (broadcasting and gossiping), in: Combinatorial Network Theory, (D.-Z. Du, D. F. Hsu, eds.), Kluwer Academic Publishers, 1995, 125-212.

10. Pelc, A., Fault tolerant broadcasting an gossiping in communication networks, Networks 26 (1996), 143-156.

11. Santoro, N., Widmayer, P., Distributed function evaluation in the presence of transmission faults, in: SIGAL'90, LNCS 450, Springer Verlag, Berlin, 1990, 358-369. 